\title{
INVESTIGACIÓN/RESEARCH
}

\section{PERFIL DE ENTRADA DE LOS FUTUROS ALUMNOS DE COMUNICACIÓN EN LAS UNIVERSIDADES DE MADRID, EN 2010/2012: LA REAFIRMACIÓN DE LOS RASGOS}

\author{
Miguel Ángel Ortiz-Sobrino ${ }^{1}$ : Universidad Complutense de Madrid. España \\ amortiz.@ccinf.ucm.es
}

Dolores Rodríguez-Barba: Universidad Complutense de Madrid. España lolar@ccinf.ucm.es

María José Pérez- Serrano: Universidad Complutense de Madrid. España mariajoseperezserrano@pdi.ucm.es

\section{RESUMEN}

En el transcurso del "II Congreso Sociedad Digital: Espacios para la Interactividad y la inmersión", celebrado en Madrid, se presentó un avance de los resultados De las investigaciones llevadas a cabo en 2010 y 2011, entre alumnos que querían cursar algunos de los Grados en Comunicación en las Universidades de Madrid, para determinar su perfil de entrada. La muestra fue extraída del conjunto de participantes en una experiencia educativa denominada Summer Media School que había sido organizada por la Fundación FIDES, en Madrid. La investigación fue realizada por un grupo de investigadores del Departamento de Periodismo IV de la Universidad Complutense de Madrid. Su objetivo era elaborar un perfil de entrada de los futuros alumnos de esos Grados y los diferentes motivos que les impulsaban a realizar estos estudios. Sin embargo, la investigación no perdía de vista, como objetivo, la relación de los futuros estudiantes de Comunicación con los media, cómo les influyen, sus relaciones de consumo y sus expectativas profesionales y universitarias. Hasta ahora, las pocas investigaciones realizadas en este sentido en España habían tenido como objeto - en su mayoría- los estudiantes que ya estaban cursando los Estudios de Comunicación. En este caso, la singularidad de estos dos trabajos radica en el hecho de que la muestra la constituían alumnos candidatos a

\footnotetext{
${ }^{1}$ Autor Correspondiente Miguel Ángel Ortiz-Sobrino: Profesor Contratado Doctor. Ciencias de la Información. Universidad Complutense de Madrid. España

Correo: amortiz.@ccinf.ucm.es
} 
cursar los Grados de Comunicación. A continuación, se presentan las principales conclusiones de la investigación realizada en 2011 y en qué medida el perfil de entrada de estos alumnos se ha modificado de un año a otro.

PALABRAS CLAVE: Comunicación - Medios - Alumnos - EEES - Alfabetización Audiovisual

\title{
ACCESS PROFILE OF THE STUDENTS OF UNIVERSITIES OF MADRID IN 20/2012: THE REAFIRMATION OF THE FEATURES
}

\begin{abstract}
During the celebration in Madrid of "Digital Society Congress: Spaces for interactivity and immersion", it was presented a preview of the results of two investigations carried out in 2010 and 2011, about students who wanted to study some of the communication degrees in one of universities of Madrid, to determine their access profile. The sample was obtained from all participants in an educational experience called Media Summer School, which was organized by FIDES Foundation in Madrid. The research was conducted by a group of investigators of IV Journalism Department of the Complutense University of Madrid. Its aim was to develop an access profile of the future students in those degrees and the different motivations which impelled to perform these studies. However, research has not lost sight of the aim of studying the relationships of the future students of communication with the media, how it influences them, consumer relationships and their professional and academic expectations. Until now, little research in this direction in Spain had been intended and, mainly, about who were already enrolled in Communication Studies. In this case, the uniqueness of these two works lies in the fact that the sample was selected from candidates' students for Communication Degrees. Now, it presents the main findings of research conducted in 2011 and to what extent the profile has changed from one year to another.
\end{abstract}

KEY WORDS: Communication - Media - Students - EEES - Media literacy 


\section{INTRODUCCIÓN}

\subsection{La Summer Media School y la investigación sobre el perfil de entrada de los alumnos en los Grados en Comunicación de las Universidades madrileñas, en el contexto europeo: 2010-2012.}

En los últimos años de la primera década y el umbral de la segunda del nuevo siglo, la Unión Europea ha venido manifestando una especial preocupación por la relación de los jóvenes con las TIC y su alfabetización mediática, en especial con las denominadas "pantallas": Internet, televisión, móvil. En ese sentido, la recomendación europea pasa por la recomendación de que cada país ponga un especial empeño en la alfabetización multimedia de su población infantil y juvenil.

Con motivo de esa recomendación, en marzo de 2010 y 2011, la Fundación FIDES y la Universidad Francisco de Vitoria de Madrid, convocaron la segunda y tercera edición de la Summer Media School, una experiencia educativa configurada con el formato de un curso de verano dirigido a futuros estudiantes de algunos de los Grados en Comunicación impartidos en las Universidades de la Comunidad de Madrid, tanto públicas como privadas. . Su objetivo era elaborar un perfil de entrada de los futuros alumnos de esos Grados y los diferentes motivos que les impulsaban a realizar estos estudios, así como su relación con los "media". Los alumnos que participaron en esta edición provenían de diferentes Comunidades Autónomas.

Los 300 alumnos asistentes al curso, en la segunda edición, y los 160 alumnos de la tercera, lo hicieron en su condición de becarios, tras haber sido seleccionados en un concurso patrocinado y promovido por la Fundación FIDES y la Universidad Francisco de Vitoria, dirigido a futuros estudiantes de Comunicación en la Comunidad de Madrid. Estos alumnos aspiraban a ocupar una de las más de cuatro mil plazas en las diferentes Facultades de Comunicación de la Comunidad de Madrid. Según datos del Espacio Madrileño de Educación Superior, durante el curso 2009/10 y 2010/11 se matricularon en Madrid más de 4000 alumnos distribuidos entre las diferentes Universidades - públicas o privadas- en los que se impartían Grados o Licenciaturas de Comunicación (Ortiz y Rodríguez Barba, 2011:1-3).

Los participantes en la segunda y tercera edición de la Summer Media School fueron seleccionados entre un grupo de algo más de tres mil alumnos de Bachillerato y - en menor medida- otros procedentes de Ciclos Superiores de Formación Profesional, candidatos a cursar estudios de Comunicación en la Comunidad de Madrid, que participaron en el concurso convocado por la Fundación FIDES y la Universidad Francisco de Vitoria.

A los alumnos seleccionados, que participaron en la Summer Media School, se les pasó un cuestionario de 34 preguntas. Otros 22 alumnos, cuya selección se realizó atendiendo a una distribución equitativa entre sus preferencias por alguno de los Grados en Comunicación -Periodismo, Comunicación Audiovisual o Publicidadasistieron a un focus group dirigido y coordinado por profesores de la Universidad 
Complutense. El objetivo, tanto del cuestionario como del focus group, era obtener una serie de datos/respuestas para conseguir datos sobre el perfil de entrada de los futuros alumnos de Comunicación de las universidades madrileñas. La base de la muestra tomada de un colectivo de más de tres mil futuros alumnos representa, por ejemplo en la segunda edición, cerca del $10 \%$ de los alumnos que finalmente se matriculan en algunas de la Facultades de Comunicación de Madrid, lo que puede considerarse como una buena representación del total de los alumnos que han iniciado sus estudios de Comunicación en la Comunidad de Madrid en el curso 2010/11. o que han hecho en 2011/2012 (Ortiz, Rodríguez a \& Pérez, 2011, pp. 1-2).

Las investigaciones, cuyos principales resultados se abordan a continuación, fueron realizadas por un grupo de investigación (1) perteneciente al Departamento de Periodismo IV de la Universidad Complutense de Madrid.

\section{METODOLOGÍA}

La presente investigación fue realizada partir del empleo de los métodos teóricos: de lógico-abstracto para poder identificar las características esenciales del objeto de investigación y realizar un análisis en el contexto en las universidades de Madrid.

Se ha empleado el método de análisis documental para la consulta de reportes estadísticos acerca de los estudiantes de las Universidades. Del mismo modo para el análisis y discusión de los resultados se empleó de los métodos de la investigación cualitativa el naturalista descriptivo.

Entre los métodos empíricos que se emplearon fueron: la observación y la medición, mientras

\subsection{El perfil de la muestra.}

Si se observan los resultados de las dos investigaciones realizadas en relación con el perfil de la muestra se extrae una conclusión clara que ratifica la observación ecológica y empírica de la realidad de las aulas de las diferentes Facultades de Comunicación: el predominio de las mujeres en las aulas. Así, en la investigación realizada en 2010, entre los candidatos a estudiar el Grado de Periodismo, el 48\% eran mujeres y el $24 / \%$ eran hombres. De los alumnos que se interesaban por cursar los estudios de Comunicación Audiovisual, el $78 \%$ eran mujeres y el $24 \%$ hombres. Similar representación se encontraba entre los que optaban por cursar los estudios de Publicidad y Relaciones Publicas, con el $80 \%$ de mujeres y un $20 \%$ de hombres.

En cambio, en la muestra de 2011, entre los candidatos a estudiar Comunicación Audiovisual el $36 \%$ eran hombres y el $64 \%$ mujeres. Entre los candidatos a estudiar Periodismo, sólo el $17 \%$ eran hombres frente al 83\% que eran mujeres. En cuanto a los candidatos a estudiar Publicidad, el $85 \%$ eran mujeres y el resto hombres.

Como el objeto de estudio era elaborar un retrato robot con los principales rasgos de los perfiles de los futuros alumnos de los Grados de Comunicación en la Comunidad 
de Madrid, es razonable que en la muestra analizada hubiera una presencia mayoritaria de alumnos que provenían de centros de formación de la Comunidad Autónoma madrileña. En este sentido, en las dos investigaciones realizadas se detectó una amplia mayoría de alumnos que venían de la propia Comunidad de Madrid y, a muy larga distancia, alumnos procedentes de otras Comunidades Autónomas: Comunidad Autónoma de Valencia, Comunidad Autónoma de Andalucía etc... En 2010, entre los aspirantes a los estudios de Periodismo, el $42 \%$ eran de la Comunidad de Madrid, el $92 \%$ en el caso de los aspirantes a cursar el Grado en Comunicación Audiovisual también eran madrileños, lo mismo que casi el $50 \%$ de los candidatos al primer curso de Publicidad y Relaciones Públicas. Porcentajes similares se repetían, en 2011, respecto a la procedencia de los candidatos: en el caso de los candidatos a estudiar Periodismo representaban casi el 71\%, alrededor del $83 \%$ de los futuros alumnos de Publicidad venían de la Comunidad Autónoma de Madrid, y un 79\% de los candidatos a los estudios de Comunicación Audiovisual también provenían de esta Comunidad Autónoma (Ortiz, Rodríguez Barba y Pérez, 2011:1-2).

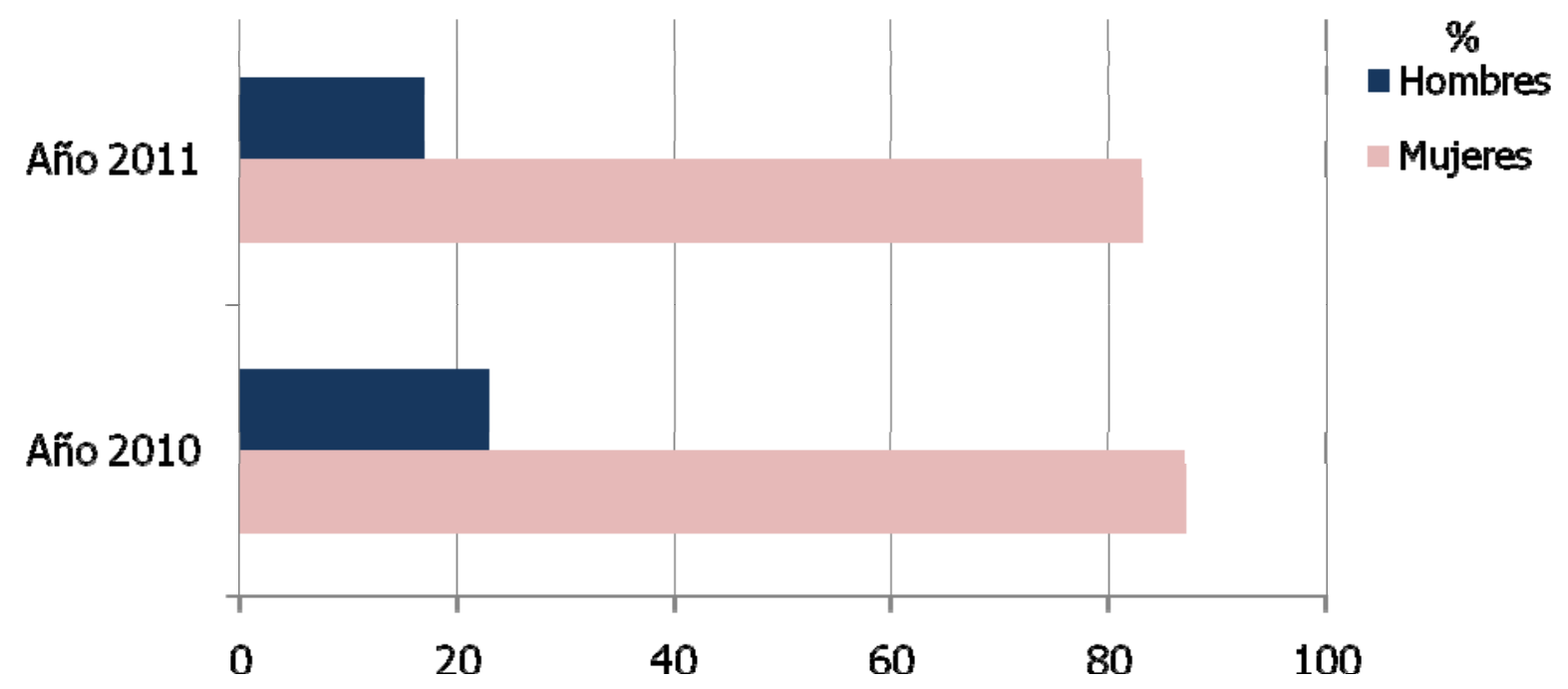

Figura 1. Perfil de la muestra, según género expresada en porciento

\subsection{Objetivos del trabajo de campo.}

El trabajo de campo pretendía dibujar un perfil de entrada de los futuros alumnos de las Facultades de Comunicación que asistieron a la Summer Media School. Fundamentalmente, en relación con aspectos relacionados con su motivación/vocación, su relación con los medios de comunicación, sus referentes profesionales, así como las expectativas y principales demandas en el tipo de formación que esperan recibir en la Universidad.

\subsection{Objetivos de la investigación}


a)

Elaborar un perfil de entrada a los Grados de Comunicación resaltando los aspectos motivacionales y vocacionales

b)

percibida por los aspirantes de la enseñanza de la

c) Universidad.

Detectar la imagen

d) demandas en el tipo de formación que se espera recibir.

Comunicación en la

Definir expectativas $y$

e)

comunicación que son referentes profesionales

f)

condicionan el consumo de los futuros profesionales de la comunicación.

g)

futuros profesionales con los medios tomados como audiencia.

h)

uso real de la TIC en sus relaciones personales.

Determinar los medios de

Determinar si los medios

Establecer la relación de los

Determinar un índice de

\subsection{La dimensión metodológica: la cuantificación y el focus group.}

En las dos investigaciones llevadas a cabo se han utilizado las mismas metodologías y las mismas herramientas de recogida de datos (Figura 1). Siguiendo la recomendación de diferentes autores (Martín González 2010: 34-39). La metodología cuantitativa de la investigación se ha basado en la utilización de un cuestionario de treinta y cuatro preguntas multirespuesta en el que se brindaba al alumno la posibilidad, en algunos casos, de graduar su respuesta. Este cuestionario fue suministrado y respondido por los 300 alumnos asistentes a la Summer Media School, en 2010, y a 160 alumnos que asistieron a la edición de 2011, base de la muestra de estas dos investigaciones. Cada uno de los alumnos respondió el cuestionario introduciendo sus respuestas en un ordenador con una plantilla de preguntas y respuestas para su posterior tratamiento informático. En las dos investigaciones, el alumno contó con la asistencia del Departamento de Informática de la Fundación FIDES y un instructivo para la cumplimentar el cuestionario que fue elaborado por los responsables de la investigación.

Al mismo tiempo, de los casi 460 alumnos a los que se les suministró el cuestionario, se seleccionaron 22 alumnos para hacer un focus group cualitativo que representaban proporcionalmente a los alumnos que optaban a los diferentes Grados. En este focus group se demandó al alumno una verbalización sobre los aspectos más relevantes que se habían planteado en el cuestionario utilizado en la investigación cuantitativa. Posteriormente, los verbatings obtenidos en el focus group fueron transcritos para reforzar y matizar los resultados de la encuesta, de acuerdo a este modelo de investigación (Castaño 2002, pp. 5-40). 


\section{METODOLOGÍA 2010-2011}

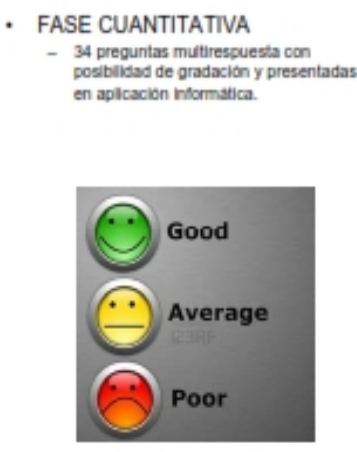

- FASE

CUALITATIVA

-22 aspirantes,

seleccionados

proporcionalmente a la

distribución de la

muestra por género y

estudios preferidos.

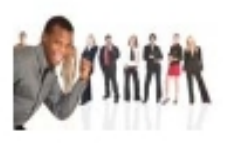

Figura 2. Metodología 2010-2011

La información obtenida a través de estas metodologías y herramientas es la base de las conclusiones de esta investigación que ahora se presentan.

\subsection{Referencias para la investigación}

Tal y como se pone de manifiesto en el número 114 de la revista Vivat Academia y en una de las Comunicaciones de II Congreso Sociedad Digital: Espacios para la Interactividad y la inmersión, son varios los estudios que se han llevado a cabo en alguno de los aspectos que aborda la presente investigación aunque, como más arriba se ha dicho, las características de la muestra la dota de cierta singularidad. En este sentido, se pueden encontrar estudios en el ámbito de la psicología y la motivación (Núnez Pérez y otros, 2009:16) y en relación con las expectativas de los universitarios (Pichardo Martínez \& et al-, 2007, p. 6; Garduño, 2006, p 4).

No menor es el interés de los investigadores por el consumo y la relación de los jóvenes con los medios de comunicación. Algunas revistas científicas como Comunicar (Ortiz 2008:10) o Telos, han recogido a lo largo de su trayectoria los resultado de gran cantidad de investigaciones realizadas a tenor de la relación de los niños y jóvenes con las pantallas (Ortiz \& Rodríguez, 2011, pp. 1-3). Pero probablemente una de las experiencias más cercanas a la investigación que ahora se presenta fue llevada a cabo en el curso 2001-2002 en la Escuela de Biblioteconomía y Documentación de la UCM y que es recogida por el profesor Tejada (Tejada, 2003, pp. 195-211), en la que se hizo una experiencia similar entre 250 alumnos matriculados en el primer curso de la Escuela. A pesar de que el número es cuantitativamente similar a la muestra de la investigación cuyas principales conclusiones se presentan en este documento, sin embargo, aquella tenía un cierto sesgo ya que no analizaba y comparaba las motivaciones de sus estudiantes con las de los alumnos de otros títulos de Comunicación (Ortiz, Rodríguez Barba y Pérez,

2011, pp.1-2). 


\section{ANÁLISIS Y DISCUSIÓN}

\subsection{Principales resultados de la Investigación.}

Las principales conclusiones extraídas de las dos investigaciones se han centrado en los tres ítems que mejor contribuyen a la construcción de perfil de entrado objeto de los dos trabajos de campo: la vocación, la relación con los medios y sus expectativas universitarias.

Extraer conclusiones sobre la vocación era importante porque permite establecer el grado de conocimiento de los futuros alumnos sobre los medios de comunicación, y en qué medida no estaban condicionados en su elección por aspectos"lúdicos" o de cierto snobismo de la profesión. Respecto a su relación con los medios interesaba especialmente saber donde encuentran sus referencias profesionales y -sobre todo su relación con los medios como fuentes de información. Finalmente, importaba extraer las expectativas que futuros alumnos tenían puestas en su formación universitaria, sobre todo en aquellos aspectos que indicaran si ven la profesión desde una dimensión más práctica, simplemente de oficio, o como una formación que además contribuya a la búsqueda de la verdad y a la contextualización de los hechos. En este sentido, de las dos investigaciones se extraen los siguientes resultados.

La vocación de los futuros comunicadores tiene cierta consistencia y se aleja la idea de un estudiante de Comunicación fruto de un sueño de juventud. Los futuros estudiantes de los Grados en Comunicación son relativamente vocacionales, no se inclinan por los estudios de Periodismo por el efecto moda y creen que con su trabajo pueden contribuir a un mundo mejor. Sin embargo, los datos porcentuales en relación a la vocación varían dependiendo de los diferentes Grados: Periodismo, Comunicación Audiovisual o Periodismo.

\subsubsection{Nuevos estudiantes de Periodismo.}

En 2010, más del 33\% de los futuros estudiantes del Grado de Periodismo creía, de una manera clara, que ésta era su verdadera vocación, aunque no son muy optimistas respecto a sus salidas profesionales. El efecto moda no condicionaba mucho a los futuros estudiantes de Periodismo: el 65\% reconocía que no estudiaría Periodismo porque sea una profesión de moda o popular y sólo el $18 \%$ se consideraba con algún tipo de influencia en este sentido. Es de destacar que sólo el

$10 \%$ de la muestra pensaba que el trabajo como periodista le haría ganar popularidad.

Respecto a la responsabilidad social y su dimensión ética, los futuros periodistas encuestados creían que el Periodismo puede contribuir a la construcción de un mundo mejor: el $40 \%$ lo afirmaba con cierta rotundidad y el $30 \%$ opinaba que, de alguna forma, el periodismo puede ser una herramienta que puede contribuir a la transformación del mundo. 
En el trabajo de campo llevado a cabo en 2011, la vocación por la profesión periodística aparece más claramente entre los encuestados: más del $46 \%$ manifiestan optar por vocación a los estudios de Periodismo. Quizá este resultado tiene relación con el hecho de que, a pesar de la crisis que atraviesan los medios de comunicación, casi un $40 \%$ de los encuestados es relativamente optimista en relación con su futuro profesional.

En este caso, el efecto moda parece que tampoco influye en su opción: más del $90 \%$ manifiesta no sentirse condicionados por el efecto moda en su elección de los estudios. En relación al año anterior, pierde fuerza la idea de que es necesario trabajar en un medio de comunicación para conseguir ser un personaje popular. Aunque en menor proporción que en 2010, los encuestados de 2011 siguen pensando que el ejercicio del Periodismo le permitirá trabajar por un mundo mejor: concretamente el $36,5 \%$ está convencido de ello.

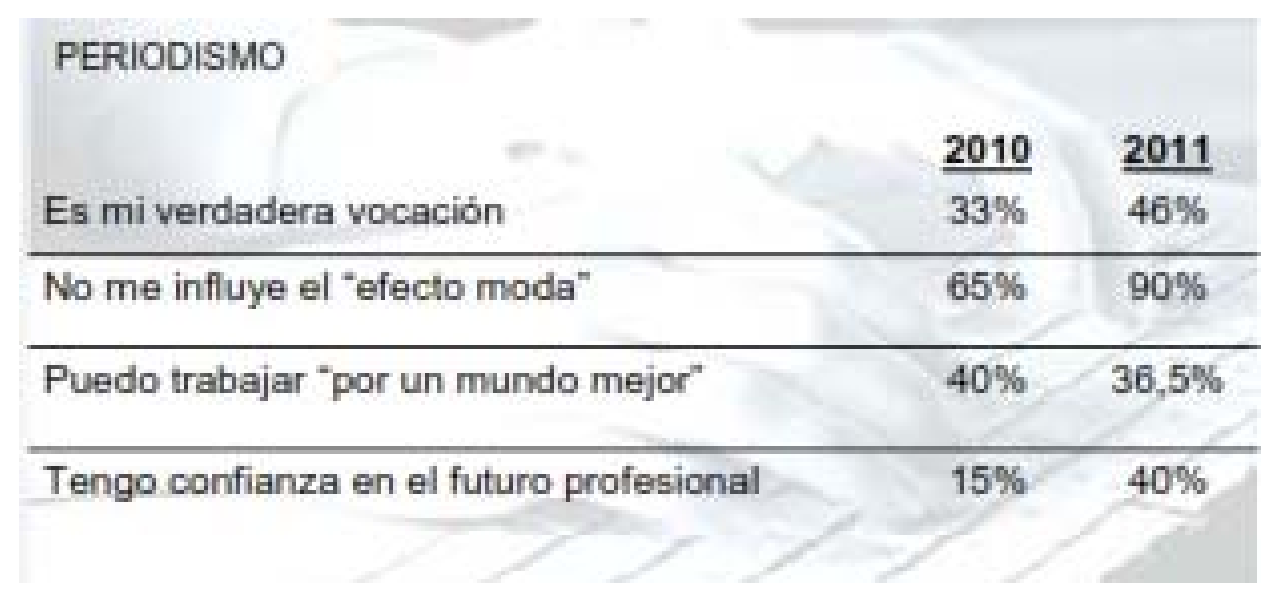

Figura 2. Resultados sobre motivación (I)

\subsubsection{Nuevos estudiantes de Comunicación Audiovisual.}

En 2010, entre los candidatos a estudiar Comunicación Audiovisual se daba uno de los mayores porcentajes de interés por los contenidos del Grado y un índice muy alto de vocación. Algo más del $78 \%$ de los encuestados consideraban que la vocación estaba detrás de la elección de estos estudios. En igual sentido, se puede hablar del interés por los contenidos audiovisuales: a más del $81 \%$ de los encuestados les interesaba bastante o mucho este tipo de contenidos y su forma de creatividad. En relación con los futuros estudiantes de Periodismo, contrasta el optimismo respecto a su futuro: casi el $40 \%$ de los encuestados creen que estos estudios les pueden conducir al mercado laboral.

Entre ellos se detectaba una fuerte convicción de que los medios audiovisuales tienen una fuerte responsabilidad social porque pueden condicionar la percepción del mundo: así lo manifiesta el $50 \%$ de los encuestados. 
En la investigación realizada en 2011, se confirmaba el alto nivel vocacional de los aspirantes a estudiar Comunicación Audiovisual, aunque con menor intensidad: algo más del $47 \%$ creía estudiar el Grado de Comunicación Audiovisual por vocación, claramente, mientras que otro $40 \%$ respondía que era su vocación con un nivel de convencimiento intermedio. Como en 2010, los datos pueden estar relacionados con sus perspectivas profesionales porque descendía considerablemente el grado de optimismo sobre su futuro profesional: menos del $5 \%$ de los encuestados eran muy optimistas respecto a su inserción profesional. También en este caso, se manifestaba un claro interés por los medios y contenidos audiovisuales: más del $78 \%$ de los encuestados manifestaban interesarles bastante o mucho los medios audiovisuales.

En cuanto a la responsabilidad social de los medios, solo el $36,5 \%$ de los que se planteaban su trabajo desde una perfectiva ético-social para transformar el mundo.

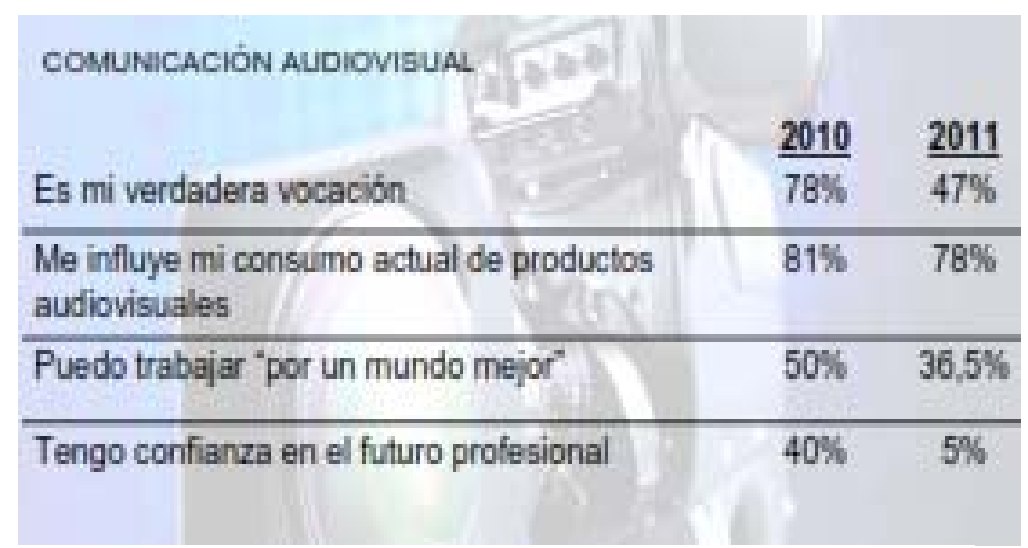

Figura 3. Resultados sobre motivación (II)

\subsubsection{Nuevos estudiantes de Publicidad y Relaciones Públicas.}

Respecto a la vocación, se detecta una cierta contradicción entre los datos arrojados en una y otra encuesta. Para los futuros alumnos del Grado de Publicidad y RRPP la vocación era, en 2010, uno de los argumentos menos valorados respecto a sus compañeros de los Grados de Periodismo y Comunicación Audiovisual: sólo un 14\% declaraba que había sido la vocación uno de los motivos fundamentales para cursar los estudios de este Grado, frente al 53\% que manifestaba tener poca o ninguna vocación. A pesar de este bajo índice de vocación, los nuevos estudiantes de Publicidad estaban en línea con las opiniones de sus colegas de Comunicación Audiovisual en relación con su futuro: el 36\% de los encuestados creía que tendrán muchas o bastantes salidas profesionales, una vez que acaben sus estudios.

Frente a los alumnos de Periodismo o Comunicación Audiovisual, para casi el $60 \%$ de los encuestados, el prestigio social fue uno de los argumentos barajados a la hora de inclinarse por esta titulación

Una conclusión importante que se extrae de la investigación es que los futuros estudiantes encuestados pensaban que estos estudios tienen una mayor proyección 
social y que más de la mitad de los encuestados pensaba que se puede contribuir a un consumo más saludable desde el ejercicio de la profesión publicitaria.

En 2011, a pesar de que los niveles de confianza respecto al futuro son similares al año anterior, los resultados arrojados por la encuesta cambian sustancialmente. Descienden los que manifiestan tener poca o ninguna vocación (38,5\%) y suben (el $33 \%$ de encuestados) los que manifiestan tener una vocación clara o muy clara por los estudios de Publicidad.

Los indicadores relacionados con la responsabilidad social de su futura profesión o con su prestigio social, también sufren variación. Baja el porcentaje de encuestados (el $43 \%$ ) que piensa que puede contribuir a mejorar la sociedad desde el ejercicio de su profesión. De igual forma, desciende el número de candidatos que han elegido estos estudios para alcanzar un prestigio social: en torno al $20 \%$ de los encuestados

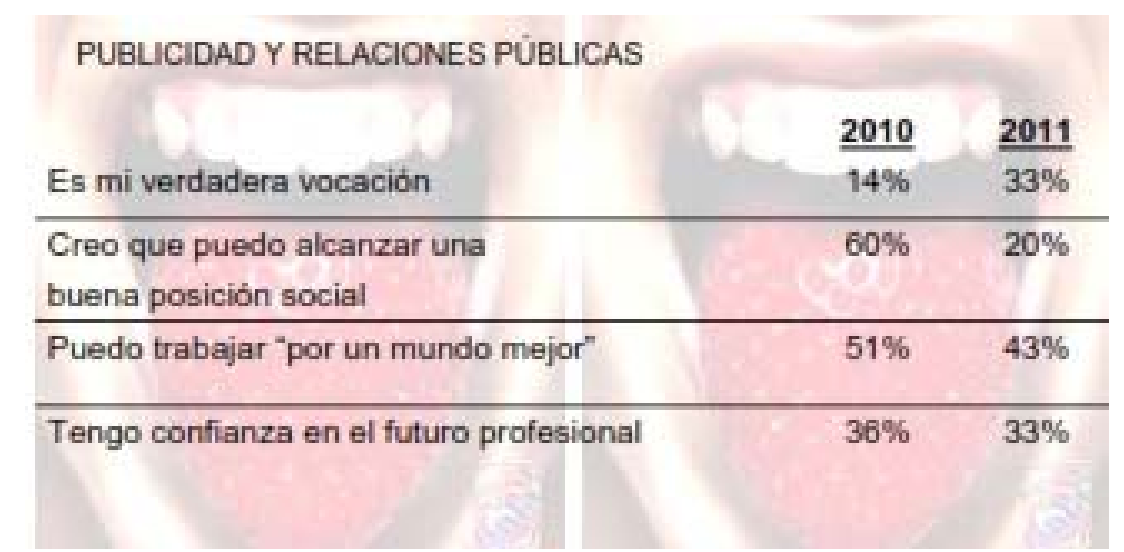

Figura 4. Resultados sobre publicidad y relaciones públicas (I)

La prensa escrita y la televisión se convierten en modelo: los referentes profesionales de los futuros comunicadores están entre los que trabajan en los medios de comunicación convencionales:

En 2010, más del $42 \%$ de los futuros profesionales de la información tenían como referencia profesional especialmente a los periodistas de la prensa escrita. Los periodistas de los medios impresos eran los que mayor credibilidad les transmitían, seguidos de los presentadores de televisión (21\%) y los de la radio (25\%). Si se tiene en cuenta que el colectivo encuestado estaba compuesto por "nativos digitales", familiarizados con el uso de la Red y las Nuevas Tecnologías, sorprende la escasa credibilidad y profesionalidad que otorgaban a los tertulianos de los programas de radio y televisión $(2 \%)$-a pesar de que las tertulias son un formato de gran implantación en los dos medios- y la poca credibilidad y profesionalidad que les transmitía la información de los blogs (5\%)

En relación con los futuros estudiantes de Comunicación Audiovisual, los profesionales del sector cinematográfico eran la referencia profesional para, prácticamente, casi la mitad de los encuestados (49\%), frente a casi el $24 \%$ cuyos referentes profesionales se encontraban en el mundo de la televisión. Los profesionales de la radio exclusivamente eran referencia para el $7 \%$ de los encuestados. Aunque lo 
realmente sorprendente es la baja presencia de los profesionales de los videojuegos que-curiosamente- solo para el $2 \%$ de la muestra son una referencia profesional.

En cuanto a los nuevos estudiantes de Publicidad, sus referentes profesionales los encuentran en la televisión (36\%) y en el cine $(26,6 \%)$, porcentajes lógicos de respuestas si se tiene en cuenta que se trata de jóvenes con un fuerte consumo audiovisual. Sin embargo, destacan los bajos porcentajes obtenidos por las referencias a otros soportes que les resultan familiares, como Internet.

Tal y como se ponen de manifiesto los autores de la investigación en otras contribuciones científicas, en 2011, ya mencionadas sobre los resultados de estas dos investigaciones ,los futuros estudiantes de Periodismo encontraban sus referencias profesionales en la televisión $(41,5 \%)$ y en la prensa escrita (36,5\%). Entre los estudiantes de Comunicación Audiovisual, sus referencias profesionales se situaban entre los profesionales de la televisión (24\%) y fundamentalmente entre los del sector cinematográfico $(47,6 \%)$ ).Para los alumnos de Publicidad, las referencias profesionales y sus modelos estaban entre los que trabajan en televisión (31,5\%), en el cine (30\%) o en el sector multimedia ( $30 \%$ ).

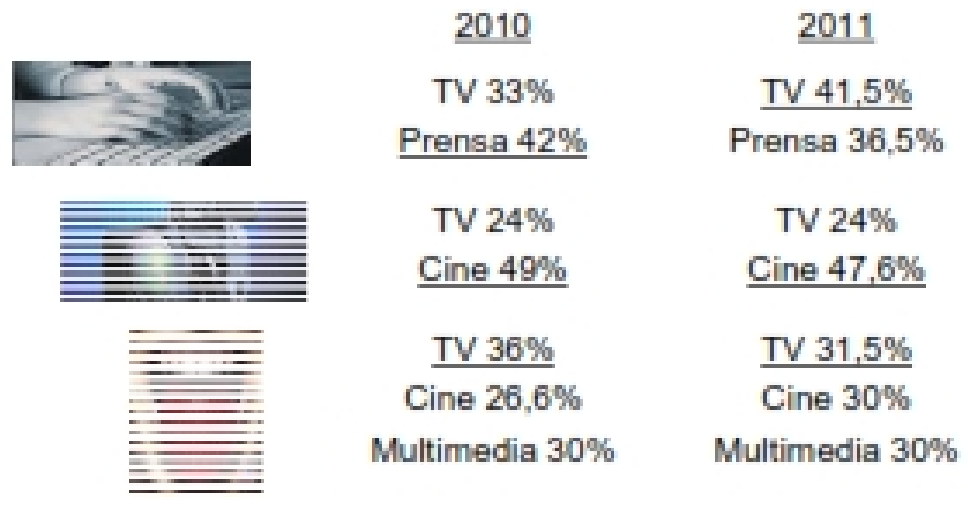

Figura 5. Resultados sobre publicidad y relaciones públicas (II)

Los nuevos Grados no acaban de posicionarse su imagen de marca: los futuros estudiantes de Comunicación no tienen una imagen excesivamente positiva sobre la enseñanza de los Grados de Comunicación.

Tal y como recoge la revista Vivat Academia, en 2010, ni siquiera el $50 \%$ de los encuestados de los nuevos alumnos de Periodismo creían que en la Universidad se forme bien a los futuros periodistas: el $42 \%$ afirmaba que la formación es buena y el $26 \%$ pensaba que sólo alguna Universidad pública o privada ofrece una buena formación.

Más optimistas que los estudiantes de Periodismo, los de Comunicación Audiovisual creían que la Universidad forma bien a los futuros comunicadores (53\%), frente a un $23 \%$ que opinaba lo contrario. El $55 \%$ de los estudiantes de Publicidad creían que la Universidad forma bien a los futuros comunicadores, aunque introducen el 
condicionante geográfico (21\%) para matizar que, dependiendo de la Comunidad Autónoma, la formación es mejor o peor (Ortiz \& Rodríguez, 2011, p. 34).

No parece que la imagen sobre los Grados mejore entre los encuestados un año después. En la encuesta realizada en 2011, la imagen de las enseñanzas de los Grados de Comunicación entre los estudiantes manifestaba un cierto estancamiento respecto al año anterior: sólo el $39 \%$ de los encuestados que se iban a matricular en Periodismo creía que la enseñanza de estos Grados de Comunicación era buena, aunque en nivel diferente dependiendo de qué Universidad. Los futuros estudiantes de Comunicación eran, como en 2010 , algo más optimistas ya que un $55 \%$ de los encuestados pensaban que la formación impartida era buena, aunque dependía de cada Universidad. En cuanto a los futuros estudiantes de Publicidad, el porcentaje de estudiantes que tenían una imagen positiva de la formación publicitaria en la universidad descendía hasta algo más del $47 \%$, condicionándolo -como en los casos anteriores- al centro y tipo de Universidad.

Los medios de comunicación condicionan el consumo de los futuros comunicadores, aunque los medios que más influyen difieren según el perfil de estudiante.

Tal y como ponían de manifiesto los autores de este texto en el número 114 de la revista Vivat Academia, en el verano de 2010 la gran mayoría de los encuestados que pensaba estudiar Periodismo creía que los medios de comunicación condicionaban sus hábitos de consumo. El $72 \%$ opinaba que, a veces, condicionaba su manera de consumir y comprar, mientras que un $14 \%$ lo afirmaba con rotundidad. Frente a esta posición, solo un $17 \%$ negaba ese condicionamiento y un $11 \%$ creía que nunca se ha sentido influido por los medios de comunicación en sus decisiones de compra. Resultaban, entonces, curiosos los datos arrojados por la encuesta respecto a los medios de comunicación que más influían en su consumo. La televisión (37\%) y las revistas (24\%) eran los soportes más influyentes. Pero sorprendía sobremanera que, entre los encuestados, se le otorgaba una incidencia prácticamente nula a las redes sociales, cuando se sabe que esta es una generación de nativos digitales en la que las redes sociales son un instrumento generalizado de comunicación( Ortiz sobrino y Rodríguez Barba, 2011: 17-18).

En el caso del grupo de Comunicación Audiovisual, se producía un cambio radical en la tendencia. En este caso era Internet $(46 \%)$ el medio que se revela ba como el de mayor influencia entre los encuestados, a muchísima distancia de la prensa (16\% ) o la televisión (4\%).

Por su parte, los futuros estudiantes de Publicidad eran conscientes de que los medios de comunicación condicionaban sus hábitos de consumo y compra, a veces $(55 \%)$ o siempre $(22 \%)$. La televisión, con un $48 \%$, y las revistas, con un $17 \%$, eran los medios considerados como más influyentes a la hora de comprar entre los futuros alumnos de Publicidad y Relaciones Públicas. 
Un año después, en el verano de 2011, se radicalizan un poco mas entre los candidatos a estudiar Periodismo, las posiciones de los que piensan que no les influye $(24 \%)$ frente a los que creen que si les condiciona (19\%).

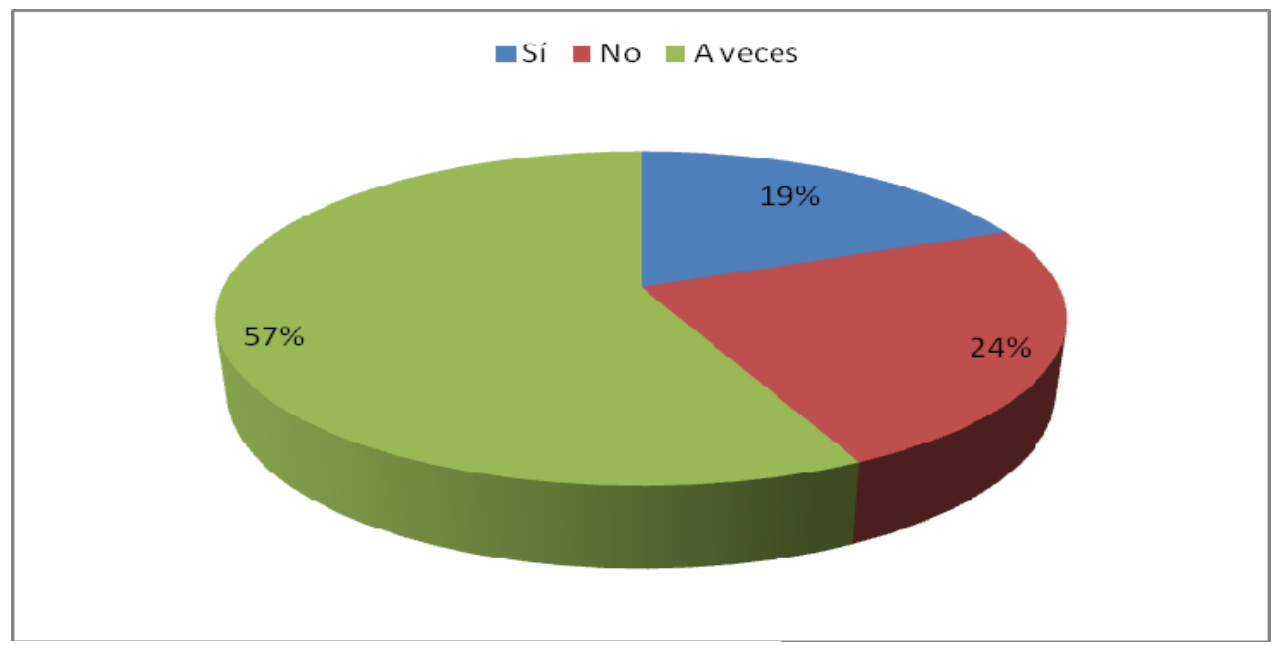

Figura 6. Alumnos de Periodismo

Resultados muy parecidos, curiosamente, arrojan las respuestas emitidas por los alumnos de Comunicación Audiovisual.

Frente a los candidatos a estudiar Periodismo o Comunicación Audiovisual, los nuevos estudiantes de Publicidad están más convencidos de su influencia en cualquier caso $(28 \%)$ o en determinadas ocasiones(67\%).

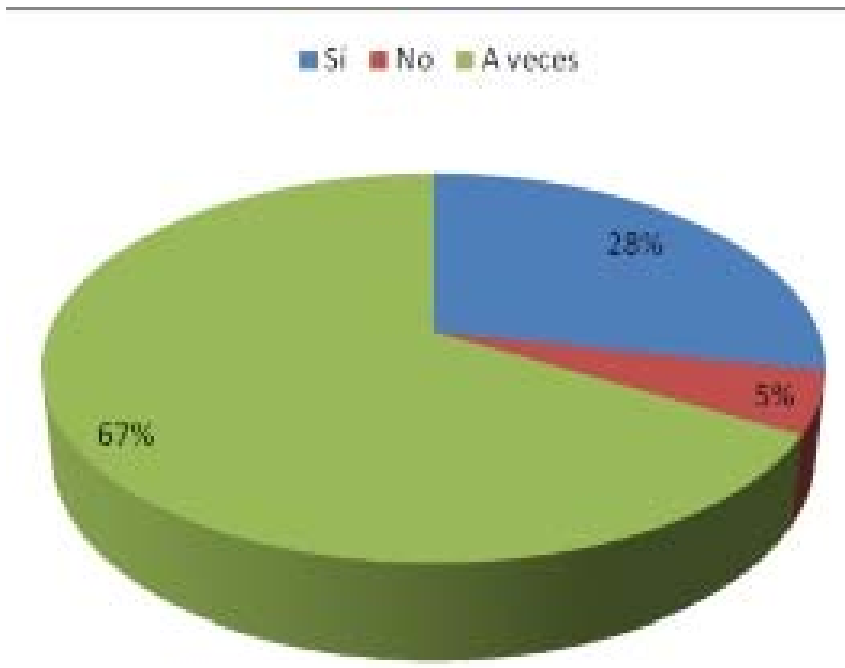

Figura 7. Alumnos de Publicidad

Expectativas sobre los contenidos de los Grados: la combinación de la teoría y la práctica es la mejor herramienta para formar a los futuros comunicadores. En 2010, casi el $44 \%$ de los encuestados opinaba que las enseñanzas de Periodismo en la 
Universidad deben ser teórico-prácticas los encuestados ponían de manifiesto que la práctica -el oficio- es tan importante como una buena formación teórica que desarrolle su capacidad de analizar y argumentar. Para ellos, era imprescindible el aprendizaje con profesionales en activo (18\%) y una buena relación de las Universidad con las empresas a través de convenios para la realización de prácticas.

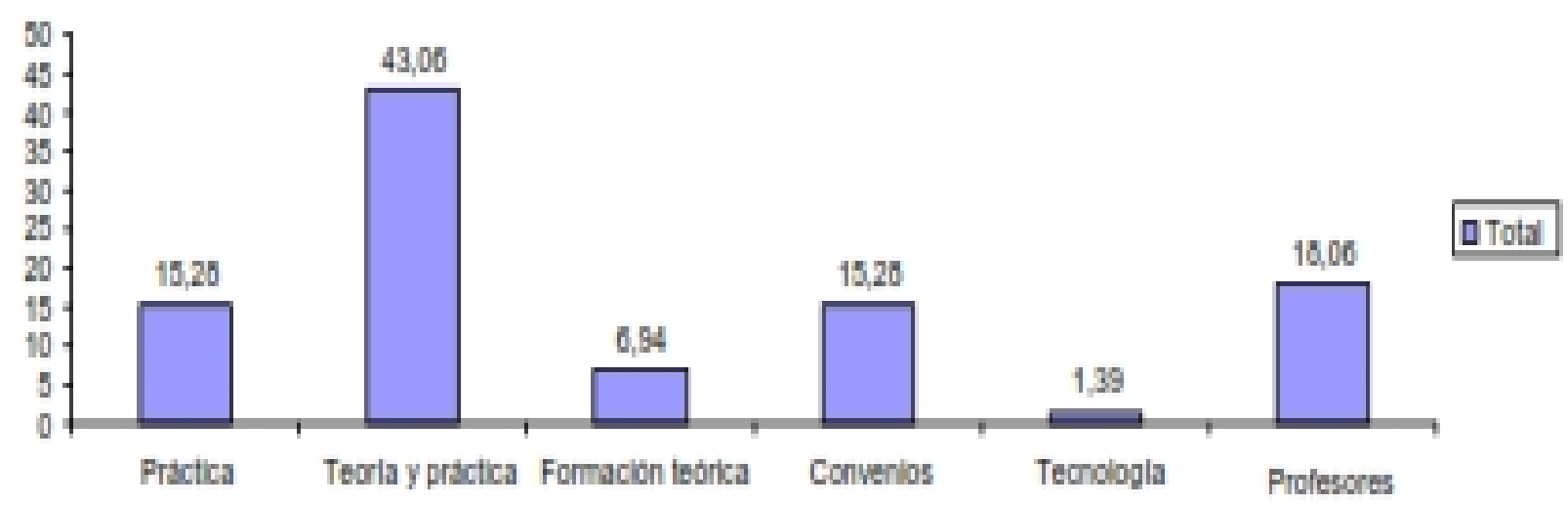

Figura 8. Ingredientes para una buena formación en los alumnos de Periodismo

Los futuros estudiantes de Comunicación Audiovisual se inclinaban por la combinación de la teoría y la práctica $(43 \%)$, frente a algo más del $20 \%$ que consideraba que debe primar sólo la práctica

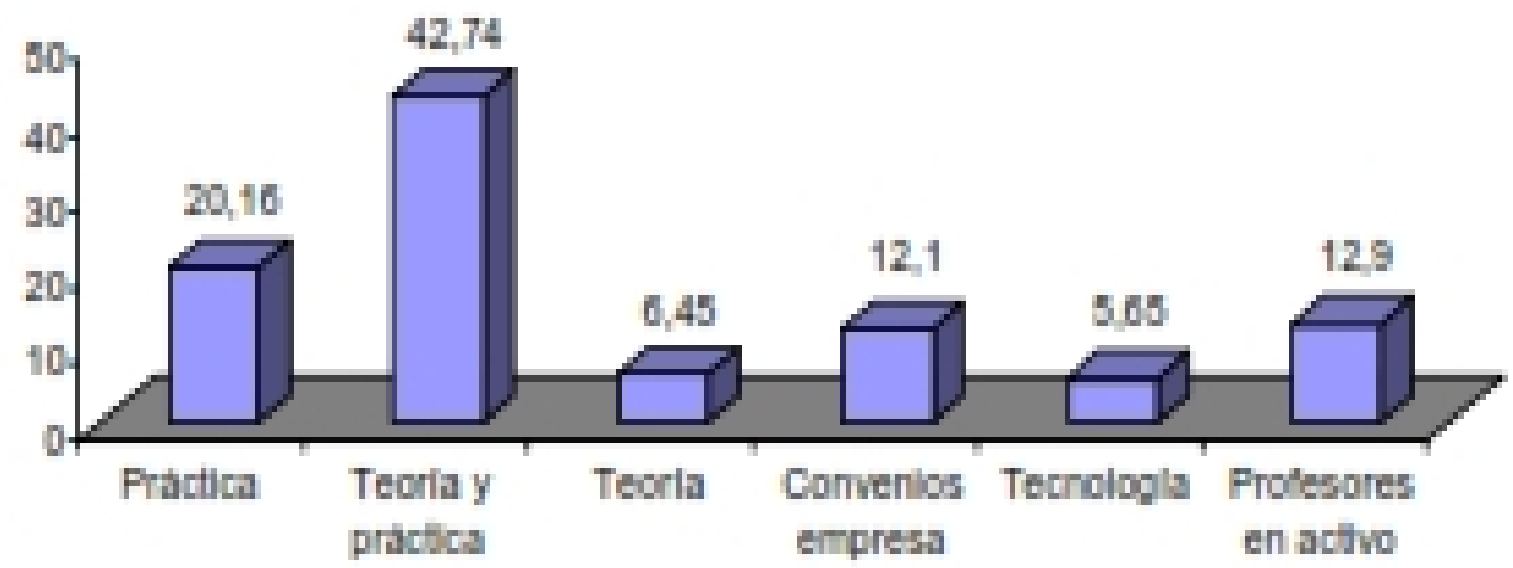

Figura 9. Ingredientes para una buena formación en Comunicación Audiovisual

Por su parte, los estudiantes de Publicidad creían que una buena combinación de teoría y práctica es la solución para la formación de los futuros comunicadores (55\%), frente a otros $(11,6 \%)$ que se inclinan sólo por la formación práctica. 


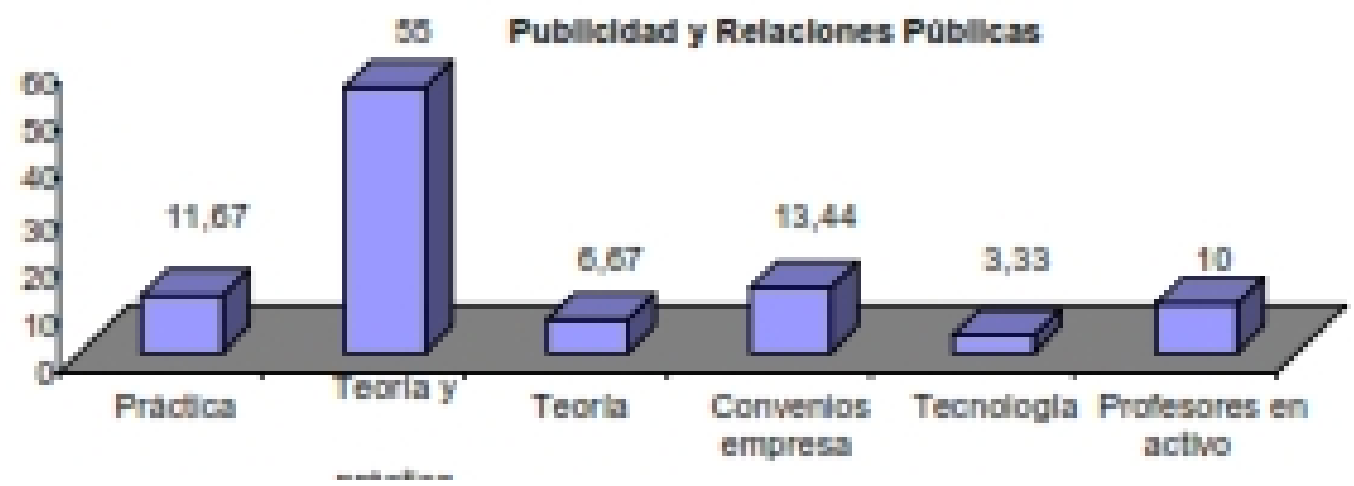

Figura 10. Ingredientes para una buena formación en Publicidad y Relaciones Públicas

Un año después, en 2011, los estudiantes de Periodismo manifestaban (46,5\%), que las enseñanzas de su carrera debería ser teórico-práctica y que los convenios con empresas para la realización de prácticas era esencial $(24,5 \%)$.

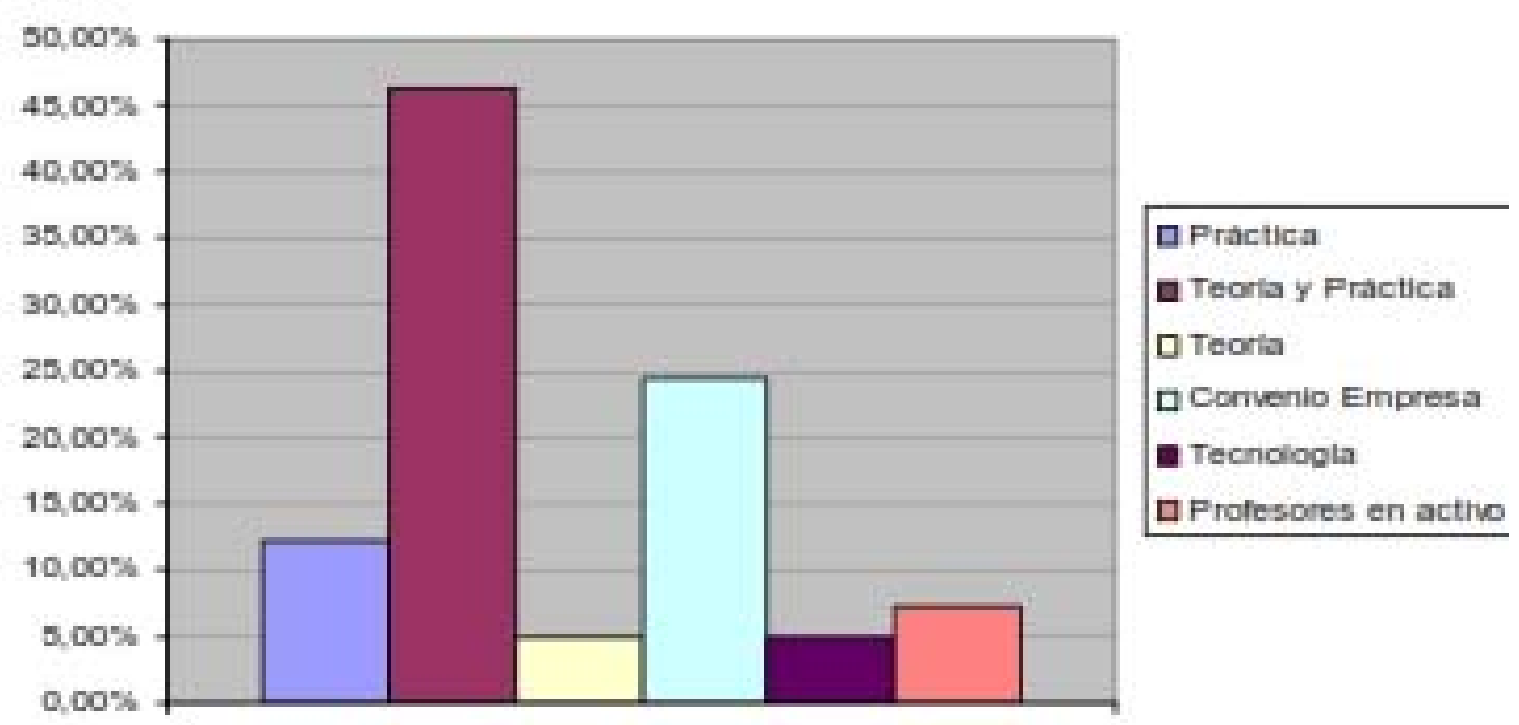

Figura 11. Ingredientes para una buena formación en Periodismo

De manera similar opinaban los futuros alumnos de Comunicación Audiovisual: para $44 \%$ de los encuestados la formación de este Grado debe ser teorico-práctica, aunque - curiosamente- desciende el número (17\%) de los que piensan que las prácticas en empresas son importantes. 


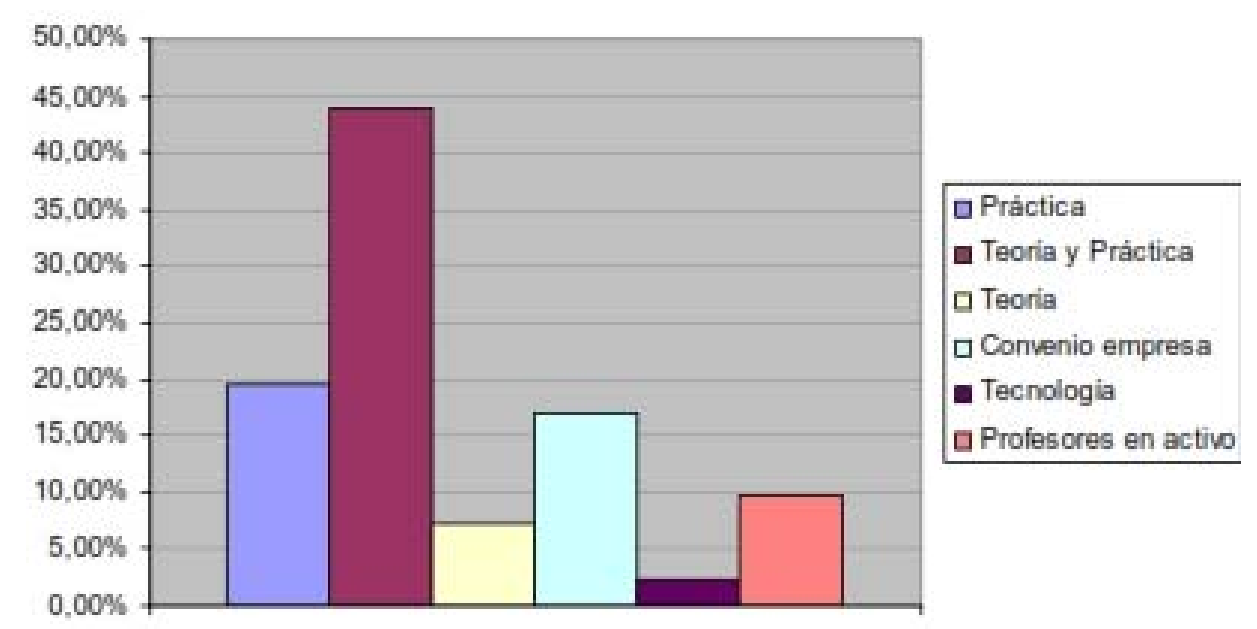

Figura 12. Ingredientes para una buena formación en Comunicación

En cuanto a los futuros alumnos de Publicidad, el $44 \%$ creía que debe ser una enseñanza teórico-práctica y le daban una relativa importancia $(17 \%)$ a las prácticas en empresas.

La enseñanza pública mantiene su imagen de calidad y sus niveles de preferencia.

En las dos investigaciones se llega de forma contundente a la conclusión de que los futuros universitarios ven la Universidad pública como centros de referencia para su formación. En 2010, la mayoría de los encuestados prefería cursar los estudios en la Universidad pública. Así lo decían los futuros estudiantes de Periodismo. También en el caso de Comunicación Audiovisual, sus estudiantes preferían la Universidad Pública (75\%). Un 65\% de los nuevos estudiantes del Grado en Publicidad también inclinaban por la universidad pública.

En el trabajo de campo realizado en 2011, la tendencia manifestada es la misma. En el caso del los futuros estudiantes de Periodismo, un 72,5 se inclina por la enseñanza pública. Un 70,5 de los alumnos de Comunicación Audiovisual tienen idéntica preferencia y algo más del $58 \%$ de los futuros estudiantes de Publicidad manifestaron que estudiarían en la Universidad pública.

Los futuros profesionales de la Comunicación encuentran en la Televisión y la Red sus principales fuentes de información.

Como se ha dicha antes al presentar las conclusiones, en cuanto a su relación con los medios interesaba especialmente saber dónde encuentran estos futuros comunicadores sus referencias profesionales y, sobre todo, su relación con los medios de comunicación como fuentes de información Según los datos obtenidos en 2010 y otros estudios realizados al respecto, se puede señalar que los medios que habitualmente utilizan los futuros comunicadores para informarse son la televisión e Internet (Tapia, 2010). Los futuros periodistas, siguen la misma tendencia general, es 
decir, tienen como medio favorito para informarse la televisión $(45,07 \%)$, seguida por Internet $(30,99 \%)$ y la prensa escrita $(11,27 \%)$.Sin embargo, ese dato contrasta con el hecho comentado anteriormente en el sentido de que sus referencias profesionales(42\% ) las encuentra, fundamentalmente, en la prensa escrita.

La respuesta de los futuros alumnos de Comunicación Audiovisual fue más que contundente en relación con este tema: el 75,61\% respondió que como fuentes de información utilizaban fundamentalmente Internet, seguido -a mucha distancia- por la televisión $(13,01 \%)$, las redes sociales, la fotografía, la radio, la telefonía móvil y otros medios. Finalmente, la mitad de los nuevos estudiantes de Publicidad tenían como medio habitual para informase, al igual que los periodistas, la televisión $(50,00 \%)$, seguido por Internet $(41,67 \%)$.

Un año después, la tendencia era similar. De los resultados obtenidos en la investigación de 2011, se extraía la conclusión de que los futuros estudiantes de Periodismo se informan -sobre todo- a través de Internet (26\%) y TV (26\%).Los de Comunicación Audiovisual se inclinaban por el uso de Internet (30,5\%) y TV (28,5\%).Por último, los futuros publicitarios, encuentran en Internet(33,5\%) y en el cine(31\%) sus fuentes informativas.

El perfil de entrada de los futuros alumno de los Grados de Comunicación mantiene los rasgos generales de su perfil en el umbral de la segunda década del nuevo siglo.

En líneas generales, puede decirse que los rasgos del perfil de entrada de los futuros alumnos de los Grados de Comunicación se mantienen y que la foto de este perfil sufre pocas alteraciones de una investigación a otra.

\section{CONCLUSIONES}

En general, los datos obtenido en las dos investigaciones confirman que los futuros alumnos de los Grados de Comunicación son relativamente vocacionales, les interesan los contenidos de los "media", creen que los medios de comunicación tiene una cierta responsabilidad social y que con su trabajo pueden contribuir a un mundo mejor: mejores hábitos de consumo, una ciudadanía mejor informada....

Los referentes profesionales de estos futuros comunicadores están entre los profesionales que trabajan en los medios de comunicación generalistas 0 convencionales. Aunque los datos difieren según la titulación elegida por los alumnos, puede concluirse que fundamentalmente es en la televisión y la prensa escrita donde encuentran sus modelos profesionales.

Los nuevos alumnos de los Grados en Comunicación apuestan más allá del mero ejercicio profesional. Creen, en este sentido, que la combinación de la teoría y la práctica es la mejor herramienta para formar a los futuros comunicadores. 
A pesar de la crisis en la estructura y mercado laboral de los medios de comunicación, los nuevos comunicadores son optimistas con su futuro profesional, aunque lo vinculan a su talento y a su preparación.

Respecto al tipo de centro preferido para su formación académica y profesional, se inclinan por titulaciones impartidas en la Universidad pública, mayoritariamente.

El Group for Research and Training of Media and Communications Management del Departamento de Periodismo IV, Empresa Informativa, de la UCM, participa en el Proyecto I+D+i CSO2008-05125 (Análisis crítico del sistema de medios: credibilidad e pacto en la ciudadanía) del Ministerio de Ciencia e Innovación, del que el prof. Farias Batlle es el IP.

Entre los resultados de este proyecto está el Informe Anual de la Profesión Periodística de la APM. Asimismo, dichos investigadores (los profesores Miguel Ángel Ortiz Sobrino (como IP), Dolores Rodríguez Barba, Manuel Fernández Sande, Fernando Peinado Miguel, María J osé Pérez Serrano y Carmen González Román) son los responsables de la investigación de referencia de esté artículo que lleva por título Perfil de entrada de los alumnos de Comunicación en la Universidad de Madrid y es el resultado del proyecto OTRI de la Universidad Francisco de Vitoria para la Fundación FIDES (2010).

Además son coautores de diferentes estudios sobre las empresas informativas y de comunicación y están especializados en medios impresos, radio, televisión y agencias de información. Son coautores de estudio financiado por la AEPG, Guía de publicaciones gratuitas de Madrid. Un recorrido por la prensa gratuita de la capital de España, editado por Madrid Emprende, en 2009, y han participado, también, en el Programa de Recursos Educativos en línea del Centro Nacional de Información y Comunicación Educativa (CNICE) (enero de 2002) y el Proyecto Diseño Metodológico y Desarrollo del Planteamiento didáctico de Media Prensa para el Proyecto Media (20022005) cuyo Investigador Principal, en ambos casos, es el Dr. Juan Carlos Alfeo Álvarez. En estos momentos (2011), además, se encuentran desarrollando el Proyecto número 247 de Innovación y Mejora de la Calidad Docente de la Universidad Complutense de Madrid, titulado Estudio de casos y desarrollo de trabajos prácticos en el aprendizaje de la Teoría de la Empresa Informativa.

\section{BIBLIOGRAFÍA}

Castaño, C.M. (2002). Introducción a la investigación cualitativa. Revista de Psicodidáctica (14): 5-40.

García Garduño, J.M. (2006). Motivación y expectativas para ingresar en la carrera de profesor de educación primaria: un estudio de tres generaciones de estudiantes normalistas mexicanos de primer ingreso REDIE: Revista de Investigación Educativa. 8, (2): 4 . 
MARTIN GONZALEZ, .JA. Cinco consejos en investigación cuantitativa. En revista MK № 258. 2010. Pag. 34-39.

Núñez Pérez, J.C. \& et al. (2009). Perfiles motivacionales en los estudios universitarios. Revista de Psicología y Educación, 1, (4): 15-30.

Ortiz Sobrino, M.A. (2008). Educar la mirada en la sociedad multipantallas. Revista COMUNICAR, (31): 10-13.

Ortiz Sobrino, M.A. \& Rodriguez Barba, D.(2011). El perfil de entrada de los alumnos de grado en las facultades de comunicación de las universidades de Madrid: el caso de la Summer Media School de la fundación FIDES/UFV. Vivat Academia (114)

Ortiz Sobrino,M.A. ;Rodriguez Barba,D.; \& Pérez Serrano, M. (2011) Perfil de entrada de los futuros alumnos de Comunicación en las Universidades de Madrid: relaciones con los contenidos mediáticos y motivaciones. Revista ICONO (14)

Pichardo, M.C; García, A.B.; de la Fuente, J. \& Justicia,F.(2007). Estudio de las expectativas en la Universidad: análisis de trabajos empíricos y futuras líneas de investigación. REDIE: Revista electrónica de investigación educativa. (1).

Tapia, A.; Gómez Nieto, B.; Herranz, J.M.\& Matellanes, M. (2010). Los estudiantes universitarios ante las redes sociales: cuestiones de uso y agrupamiento en estructuras elitistas o pluralistas. Vivat Academia. (113):3-4.

Tejada, C.M. (2003). Perfil, motivaciones y expectativas de los alumnos de primer curso de la escuela de Biblioteconomía y Documentación de la UCM. Revista General de Información y Documentación: 195-212.

\section{Miguel Ángel Ortiz Sobrino}

Doctor en Ciencias de la Información por la Universidad Complutense de Madrid, con la calificación de sobresaliente cum laude. Vinculado a RTVE desde 1977, ha desempeñado, entre otros, los cargos de Jefe de Programas de Radio 5, Subdirector de Emisoras Territoriales de RNE y Subdirector de Planificación de Programas de RNE. Entre el año 2000 y 2009 ha dirigido Instituto Oficial de Radio y Televisión. También ha sido Director Adjunto de Radio Televisión Madrid y Jefe de Programas de Onda Madrid. Asimismo, es Master Oficial en Humanidades por la Universidad Francisco de Vitoria y Diplomado en Investigación de Medios por A.I.M.C.

\section{Dolores Rodríguez Barba}

María Dolores Rodríguez Barba es Doctora en Ciencias de la Información. Trabaja en el Departamento de Periodismo IV de la Universidad Complutense de Madrid como profesora, en el Grado y Master Oficial de Periodismo, y como investigadora, en el ámbito de la Organización y Gestión de Empresas de Comunicación. Fuera de la 
Universidad, ha trabajado en TVE y en las áreas de programación y dirección técnica en diferentes cadenas de radio.

\section{María José Pérez Serrano}

Doctora en Ciencias de la Información por la Universidad Complutense de Madrid y MBA en la especialidad de Gestión Financiera. Su tesis doctoral ha sido merecedora del Premio Extraordinario de Doctorado del curso académico 2006-2007. Está acreditada por la ANECA como Profesor Ayudante Doctor, Profesor de Universidad Privada y Profesor Contratado Doctor. Su trayectoria y producción científica se vincula al estudio de la Empresa Informativa y, específicamente, a la concentración de medios de comunicación y su influencia en el pluralismo. 